\title{
A Pseudo-Boolean programming approach for computing the breakpoint distance between two genomes with duplicate genes
}

\author{
Sébastien Angibaud ${ }^{1}$, Guillaume Fertin ${ }^{1}$, Irena Rusu ${ }^{1}$, Annelyse Thévenin ${ }^{2}$, \\ and Stéphane Vialette ${ }^{2}$ \\ 1 Laboratoire d'Informatique de Nantes-Atlantique (LINA), FRE CNRS 2729 \\ Université de Nantes, 2 rue de la Houssinière, 44322 Nantes Cedex 3 - France \\ \{angibaud, fertin, rusu\}@lina.univ-nantes.fr \\ 2 Laboratoire de Recherche en Informatique (LRI), UMR CNRS 8623 \\ Faculté des Sciences d'Orsay - Université Paris-Sud, 91405 Orsay - France \\ \{thevenin, vialette\}@lri.fr
}

\begin{abstract}
Comparing genomes of different species has become a crucial problem in comparative genomics. Recent research have resulted in different genomic distance definitions: number of breakpoints, number of common intervals, number of conserved intervals, Maximum Adjacency Disruption number (MAD), etc. Classical methods (usually based on permutations of gene order) for computing genomic distances between whole genomes are however seriously compromised for genomes where several copies of the same gene may be scattered across the genome. Most approaches to overcoming this difficulty are based on the exemplar method (keep exactly one copy in each genome of each duplicated gene) and the maximum matching method (keep as many copies as possible in each genome of each duplicated gene). Unfortunately, it turns out that, in presence of duplications, most problems are $\mathbf{N P}$-hard, and hence several heuristics have been recently proposed.

Extending research initiated in [2], we propose in this paper a novel generic pseudo-boolean approach for computing the exact breakpoint distance between two genomes in presence of duplications for both the exemplar and maximum matching methods. We illustrate the application of this methodology on a well-known public benchmark dataset of $\gamma$-Proteobacteria.
\end{abstract}

Keywords: genome rearrangement, duplication, breakpoint distance, heuristic, pseudo-boolean programming.

\section{Introduction}

The order of genes in the genomes of species can change during evolution and can provide information about their phylogenetic relationship. Two main approaches are possible. The first one consists in using different types of rearrangement operations and to find possible rearrangement scenarios using these operations (one 
of the most common rearrangement operations is reversals, which reverse the order of a subset of neighboring genes) [11]. The second one consists in computing a (dis-)similarity measure based on the gene order and most common rearrangement operations $[15,8,4,1]$. We focus in this paper on the latter approach.

Several similarity (or dissimilarity) measures between two whole genomes have been recently proposed, such as the number of breakpoints $[15,8,4]$, the number of reversals $[8,11]$, the number of conserved intervals [6], the number of common intervals [7], the Maximum Adjacency Disruption Number (MAD) [16], etc. However, in the presence of duplications and for each of the above measures, one has first to disambiguate the data by inferring orthologs, i.e., a nonambiguous mapping between the genes of the two genomes. Up to now, two extremal approaches have been considered : the exemplar model and the maximum matching model. In the exemplar model [15], for all gene families, all but one occurrence in each genome is deleted. In the maximum matching model $[4,10]$, the goal is to map as many genes as possible. These two models can be considered as the extremal cases of the same generic homolog assignment approach.

Unfortunately, it has been shown that, for each of the above mentioned measures, whatever the considered model (exemplar or maximum matching), the problem becomes $\mathbf{N P}$-complete as soon as duplicates are present in genomes $[8,4,6,10]$; a few inapproximability results are known for some special cases. Therefore, several heuristic methods have been recently devised to obtain (hopefully) good solutions in a reasonable amount of time [5,7]. However, while it is relatively easy to compare heuristics between them, until now very little is known about the absolute accuracy of these heuristics. Therefore, there is a great need for algorithmic approaches that compute exact solutions for these genomic distances.

Extending research initiated in [2], we propose in this paper a novel generic pseudo-boolean approach for computing the exact breakpoint distance between two genomes in presence of duplications for both the exemplar and maximum matching methods. Furthermore, we show strong evidence that a fast and simple heuristic based on iteratively finding longest common subsequences provides very good results on our dataset of $\gamma$-Proteobacteria.

This paper is organized as follows. In Section 2, we present some preliminaries and definitions. We focus in Section 3 on the problem of finding the minimum number of breakpoints under the two models and we give a pseudo-boolean program together with some reduction rules. Section 4 is devoted to experimental results on a dataset of $\gamma$-Proteobacteria.

\section{Preliminaries}

From an algorithmic perspective, a unichromosomal genome is a signed sequence over a finite alphabet, referred hereafter as the alphabet of gene families. Each element of the sequence is called a gene. DNA has two strands, and genes on a genome have an orientation that reflects the strand of the genes. We represent the order and directions of the genes on each genome as a sequence of signed 
elements, i.e., elements with signs "+" and "-". Let $G_{0}$ and $G_{1}$ be two genomes. For each $x \in\{0,1\}$, we denote the label at position $i$ in $G_{x}$ by $G_{x}[i], 1 \leq i \leq n_{x}$, and we write $n_{x}$ for the number of genes in genome $G_{x}$ and $\operatorname{occ}_{x}(\mathbf{g}, i, j)$ for the number of genes $\mathbf{g}$ (and $-\mathbf{g}$ ) in $G_{x}$ between positions $i$ and $j, 1 \leq i \leq j \leq n_{x}$. To simplify notations, we abbreviate $\operatorname{occ}_{x}\left(\mathbf{g}, 1, n_{x}\right)$ to $\operatorname{occ}_{x}(\mathrm{~g})$.

In order to deal with the inherent ambiguity of duplicated genes, we now precisely define what is a matching between two genomes. Roughly speaking, a matching between two genomes can be seen as a way to describe a putative assignment of orthologous pairs of genes between the two genomes (see for example [11]). A matching $\mathcal{M}$ between genomes $G_{0}$ and $G_{1}$ is a set of pairwise disjoint pairs $\left(G_{0}[i], G_{1}[j]\right)$, where $G_{0}[i]$ and $G_{1}[j]$ belong to the same gene family regardless of the sign, i.e., $\left|G_{0}[i]\right|=\left|G_{1}[j]\right|$. Genes of $G_{0}$ and $G_{1}$ that belong to a pair of the matching $\mathcal{M}$ are said to be saturated by $\mathcal{M}$, or $\mathcal{M}$-saturated for short. A matching $\mathcal{M}$ between $G_{0}$ and $G_{1}$ is said to be maximum if for any gene family, there are no two genes of this family that are unmatched for $\mathcal{M}$ and belong to $G_{0}$ and $G_{1}$, respectively.

The above definition allows us a large degree of freedom in the choice of the matching between two genomes. Two types of matching are usually considered and specify the underlying model to focus on for computing the desired genomic distance. In the exemplar model, the matching $\mathcal{M}$ is required to saturate exactly one gene of each gene family, i.e., the size of the matching is the number of gene families. In the maximum matching model, the matching $\mathcal{M}$ is required to saturate as many genes of any gene family as possible, i.e., $\mathcal{M}$ is a matching of maximum cardinality. Let $\mathcal{M}$ be any matching between $G_{0}$ and $G_{1}$ that fulfills the requirements of a given model (exemplar or maximum matching). By first deleting non-saturated genes and next renaming genes in $G_{0}$ and $G_{1}$ according to the matching $\mathcal{M}$, we may now assume that both $G_{0}$ and $G_{1}$ are duplication-free, i.e. $G_{1}$ is a signed permutation of $G_{0}$. We call the resulting genomes $\mathcal{M}$-pruned.

Let $G_{0}$ and $G_{1}$ be two duplication-free genomes of size $n$. Without loss of generality, we may assume that $G_{0}$ is the identity permutation, i.e., $G_{0}=12 \ldots n$. We say that there is a breakpoint after gene $G_{0}[i], 1 \leq i<n$, in $G_{0}$ if neither $G_{0}[i]$ and $G_{0}[i+1]$ nor $-G_{0}[i+1]$ and $-G_{0}[i]$ are consecutive genes in $G_{1}$, otherwise we say that there is an adjacency after gene $G_{0}[i]$. For example, if $G_{0}=12345$ and $G_{1}=1-3-245$, then we have a breakpoint in $G_{0}$ after genes 1 and 3 (and hence we have an adjacency in $G_{0}$ after genes 2 and 4).

Let $G_{0}$ and $G_{1}$ be two genomes and $\mathcal{M}$ be a matching under any model (exemplar or maximum matching) between $G_{0}$ and $G_{1}$. We define $A_{\mathcal{M}}\left(G_{0}, G_{1}\right)$ and $B_{\mathcal{M}}\left(G_{0}, G_{1}\right)$ to be the number of adjacencies and the number of breakpoints between the two $\mathcal{M}$-pruned genomes.

We are now in position to formally define the optimization problem we are interested in. Given two genomes $G_{0}$ and $G_{1}$ and a model (exemplar or maximum matching), find a matching $\mathcal{M}$ between $G_{0}$ and $G_{1}$ that fulfills the requirements of the model such that the number of breakpoints between the two $\mathcal{M}$-pruned genomes is as small as possible. 


\section{An exact algorithm}

\subsection{Pseudo-boolean problem}

Minimizing the number of breakpoints between two genomes with duplications is an $\mathbf{N P}$-hard problem under the exemplar model even when $\operatorname{occ}_{0}(\mathbf{g})=1$ for all genes $\mathbf{g}$ in $G_{0}$ and $\operatorname{occ}_{1}(\mathbf{g}) \leq 2$ for all genes $\mathbf{g}$ in $G_{1}$ [8]. Consequently, the NP-hardness also holds under the maximum matching model.

The exact algorithms we define in this section attempt to take advantage of the existing solvers, and more precisely of the linear pseudo-boolean solvers, which are a generalization of the SAT solvers. To this end, we have to express our problem (with its two variants, according to the exemplar or maximum matching model) as a linear pseudo-boolean problem (or LPB problem), i.e. as a linear program [17] whose variables take 0 or 1 values. A number of generalizations of SAT solvers to LPB solvers have been proposed (Pueblo [18], Galena [9], OPBDP [3] and more). We decided to use for our tests the minisat+ LPB solver [12] because of its good results during PB evaluation 2005 (special track of the SAT Competition 2005).

Instead of directly writing a program that minimizes the number of breakpoints, we chose to write the complementary program which consists in maximizing the number of adjacencies between the two given genomes. There are two reasons for this choice. First, the constraints are simpler and less numerous in this latter case ; moreover, experimental tests moreover showed that the running time of our program is noticeably better by focusing on adjacencies. Second, it is easy to notice that minimizing the number of breakpoints and maximizing the number of adjacencies are equivalent problems under both the exemplar and maximum matching models. Indeed, according to the above notations, given a matching $\mathcal{M}$ between two genomes $G_{0}$ and $G_{1}$ we have:

$$
B_{\mathcal{M}}\left(G_{0}, G_{1}\right)+A_{\mathcal{M}}\left(G_{0}, G_{1}\right)=|\mathcal{M}|-1
$$

For the exemplar and maximum matching models, all the matchings $\mathcal{M}$ satisfying the model have the same size, and hence $B_{\mathcal{M}}\left(G_{0}, G_{1}\right)+A_{\mathcal{M}}\left(G_{0}, G_{1}\right)$ is a constant. Therefore, maximizing $A_{\mathcal{M}}\left(G_{0}, G_{1}\right)$ is equivalent to minimizing $B_{\mathcal{M}}\left(G_{0}, G_{1}\right)$.

\subsection{Maximizing the number of adjacencies}

The LPB program we propose considers two genomes with duplications and performs an $\mathcal{M}$-pruning which maximizes the number of adjacencies according to a specified model (exemplar or maximum matching). As discussed above, the resulting matching also minimizes the number of breakpoints between the two genomes. The LPB program, Program Breakpoint-Maximum-Matching, for the maximum matching model is given in Figure 1. The exemplar variant is easily obtained by performing only a few changes that are discussed subsequently. 


\section{Program Breakpoint-Maximum-Matching}

\section{Objective :}

Maximize $\sum_{0 \leq i<n_{0}} \sum_{i<j \leq n_{0}} \sum_{0 \leq k<n_{1}} \sum_{k<\ell \leq n_{1}} d(i, j, k, \ell)$

\section{Constraints :}
(C.01) $\forall 1 \leq i \leq n_{0}, \sum_{\substack{1 \leq k \leq n_{1} \\\left|G_{0}[i]\right|=\left|G_{1}[k]\right|}} a(i, k)=b_{0}(i)$
$\forall 1 \leq k \leq n_{1}, \quad \sum_{\substack{1 \leq i \leq n_{0} \\\left|G_{0}[i]\right|=\left|G_{1}[k]\right|}} a(i, k)=b_{1}(k)$

(C. 02) $\forall 0 \leq x \leq 1, \forall \mathrm{g} \in \mathcal{G}, \sum_{\substack{1 \leq i \leq n_{x} \\\left|G_{x}[i]\right|=|\mathrm{g}|}} b_{x}(i)=\min \left(\operatorname{occ}_{0}(\mathbf{g}), \mathrm{occ}_{1}(\mathbf{g})\right)$

(C.03) $\forall 0 \leq x \leq 1, \forall 1 \leq i \leq j-1<n_{x}, c_{x}(i, j)+\sum_{i<p<j} b_{x}(p) \geq 1$

(C. 04) $\forall 0 \leq x \leq 1, \forall 1 \leq i<p<j \leq n_{x}, c_{x}(i, j)+b_{x}(p) \leq 1$

(C.05) $\forall 1 \leq i<j \leq n_{0}, \forall 1 \leq k<\ell \leq n_{1}$, such that $G_{0}[i]=G_{1}[k]$ and $G_{0}[j]=G_{1}[\ell]$, $a(i, k)+a(j, \ell)+c_{0}(i, j)+c_{1}(k, \ell)-d(i, j, k, \ell) \leq 3$

(C.06) $\forall 1 \leq i<j \leq n_{0}, \forall 1 \leq k<\ell \leq n_{1}$, such that $G_{0}[i]=G_{1}[k]$ and $G_{0}[j]=G_{1}[\ell]$ $a(i, k)-d(i, j, k, \ell) \geq 0$ $a(j, \ell)-d(i, j, k, \ell) \geq 0$ $c_{0}(i, j)-d(i, j, k, \ell) \geq 0$ $c_{1}(k, \ell)-d(i, j, k, \ell) \geq 0$

(C.07) $\forall 1 \leq i<j \leq n_{0}, \forall 1 \leq k<\ell \leq n_{1}$, such that $G_{0}[i]=-G_{1}[\ell]$ and $G_{0}[j]=-G_{1}[k]$, $a(i, \ell)+a(j, k)+c_{0}(i, j)+c_{1}(k, \ell)-d(i, j, k, \ell) \leq 3$

(C.08) $\forall 1 \leq i<j \leq n_{0}, \forall 1 \leq k<\ell \leq n_{1}$, such that $G_{0}[i]=-G_{1}[\ell]$ and $G_{0}[j]=-G_{1}[k]$, $a(i, \ell)-d(i, j, k, \ell) \geq 0$ $a(j, k)-d(i, j, k, \ell) \geq 0$ $c_{0}(i, j)-d(i, j, k, \ell) \geq 0$ $c_{1}(k, \ell)-d(i, j, k, \ell) \geq 0$

(C.09) $\forall 1 \leq i<j \leq n_{0}, \forall 1 \leq k<\ell \leq n_{1}$, such that $\left\{\left|G_{0}[i]\right|,\left|G_{0}[j]\right|\right\} \neq\left\{\left|G_{1}[k]\right|,\left|G_{1}[\ell]\right|\right\}$ or $G_{0}[i]-G_{0}[j] \neq G_{1}[k]-$ $G_{1}[\ell]$

$d(i, j, k, \ell)=0$

(C.10) $\forall 1 \leq i<j \leq n_{0}$,

$$
\sum_{1 \leq k<n_{1}} \sum_{k<\ell \leq n_{1}} d(i, j, k, \ell) \leq 1
$$

\section{Domains :}

$\forall x \in\{0,1\}, \forall 1 \leq i<j \leq n_{0}, \forall 1 \leq k<\ell \leq n_{1}$, $a(i, k), b_{x}(i), c_{x}(i, k), d(i, j, k, \ell) \in\{0,1\}$

Fig. 1. Program Breakpoint-Maximum-Matching for finding the maximum number of adjacencies between two genomes under the maximum matching model. 
Program Breakpoint-Maximum-Matching considers two genomes $G_{0}$ and $G_{1}$ of respective lengths $n_{0}$ and $n_{1}$. The objective function, the variables and the constraints are briefly discussed hereafter.

\section{Variables:}

- Variables $a(i, k), 1 \leq i \leq n_{0}$ and $1 \leq k \leq n_{1}$, define a matching $\mathcal{M}: a_{i, k}=1$ if and only if the gene at position $i$ in $G_{0}$ is matched with the gene at position $k$ in $G_{1}$ in $\mathcal{M}$.

- Variables $b_{x}(i), x \in\{0,1\}$ and $1 \leq i \leq n_{x}$, represent the $\mathcal{M}$-saturated genes: $b_{x}(i)=1$ if and only if the gene at position $i$ in $G_{x}$ is saturated by the matching $\mathcal{M}$. Clearly, $\sum_{1 \leq i \leq n_{0}} b_{0}(i)=\sum_{1 \leq k \leq n_{1}} b_{1}(k)$, and this is precisely the size of the matching $\mathcal{M}$.

- Variables $c_{x}(i, j), x \in\{0,1\}$ and $1 \leq i<j \leq n_{x}$, represent consecutive genes according to the matching $\mathcal{M}: c_{x}(i, j)=1$ if and only if the genes at positions $i, j$ in $G_{x}$ are saturated by $\mathcal{M}$ and no gene at position $p, i<p<j$, is saturated by $\mathcal{M}$.

- Variables $d(i, j, k, \ell), 1 \leq i<j \leq n_{0}$ and $1 \leq k<\ell \leq n_{1}$, represent adjacencies according to the matching $\mathcal{M}: d(i, j, k, \ell)=1$ if and only if (i) either $\left(G_{0}[i], G_{1}[k]\right)$ and $\left(G_{0}[j], G_{1}[\ell]\right)$ are two edges of $\mathcal{M}$, or $\left(G_{0}[i], G_{1}[\ell]\right)$ and $\left(G_{0}[j], G_{1}[k]\right)$ are two edges of $\mathcal{M}$, (ii) $G_{0}[i]$ and $G_{0}[j]$ are consecutive in $G_{0}$ according to $\mathcal{M}$, (iii) $G_{1}[k]$ and $G_{1}[\ell]$ are consecutive in $G_{1}$ according to $\mathcal{M}$.

\section{Objective function:}

The objective of Program Breakpoint-Maximum-Matching is to maximize the number of adjacencies between the two considered genomes. This objective reduces in our model to maximizing the sum of all variables $d(i, j, k, \ell)$.

\section{Constraints:}

Assume $x \in\{0,1\}, 1 \leq i<j \leq n_{0}$ and $1 \leq k<\ell \leq n_{1}$.

- Constraint (C.01) ensures that each gene of $G_{0}$ and of $G_{1}$ is matched at most once, i.e., $b_{0}(i)=1$ (resp. $b_{1}(k)=1$ ) if an only if gene $i$ (resp. $k$ ) is matched in $G_{0}$ (resp. $G_{1}$ ) ; see Figure 2 for an illustration. Moreover, the matching is possible only between genes in the same family. It is worth noticing here that we do not specifically ask that $a(i, k)=0$ when $i$ and $k$ concern genes belonging to different families. This is simply not necessary.

- Constraint (C.02) defines the model (i.e. the maximum matching model, in this case). For each gene family $\mathbf{g}$, one must have a single matched gene for the exemplar model and $\min \left(\mathrm{occ}_{0}(\mathbf{g}), \mathrm{occ}_{1}(\mathbf{g})\right)$ matched genes for the maximum matching model (see Figure 2).

- Constraints in (C.03) and (C.04) express the definition of consecutive genes, thus fixing the values of the variables $c_{x}$. The variable $c_{x}(i, j)$ is equal to 1 if and only if there exists no $p$ such that $i<p<j$ and $b_{x}(p)=1$. Again, it is worth noticing that the constraints do not force the variables $c_{x}(i, j)$ to have exactly the values we intuitively wish according to the abovementioned 
interpretation. Here, we accept that $c_{x}(i, j)=1$ even if the gene at position $i$ or $j$ is not matched. However, this will pose no problem in the sequel.

- Constraints in (C.05) to (C.10) define variables $d$. In the case where $G_{0}[i]=$ $G_{1}[k]$ and $G_{0}[j]=G_{1}[\ell]$, constraints (C.05) and (C.06) ensure that we have $d(i, j, k, \ell)=1$ if and only if all variables $a(i, k), a(j, \ell), c_{0}(i, j)$ and $c_{1}(k, \ell)$ are equal to 1 . In the case where $G_{0}[i]=-G_{1}[\ell]$ and $G_{0}[j]=-G_{1}[k]$, constraints (C.07) and (C.08) ensure that we have $d(i, j, k, \ell)=1$ if and only if all variables $a(i, \ell), a(j, k), c_{0}(i, j)$ and $c_{1}(k, \ell)$ are equal to 1 . Constraint (C.09) fixes the variable $d(i, j, k, \ell)$ to 0 if none of the two cases above holds. Constraint (C.10) requires to have at most one adjacency for every pair $(i, j)$. See Figure 3 for a simple illustration.

$$
\begin{aligned}
& \text { Genome } G_{0} \quad b_{0}(i)=1 \\
& G_{0}[1] G_{0}[2] \cdots \ldots . . . G_{0}[i-1] G_{0}[i] G_{0}[i+1] \ldots \ldots \ldots . . G_{0}\left[n_{0}\right] \\
& \left\{\begin{array}{c}
\left|G_{0}[i]\right|=\left|G_{1}\left[k_{1}\right]\right| \\
\vdots \\
\left|G_{0}[i]\right|=\left|G_{1}\left[k_{j}\right]\right| \\
\vdots \\
\left|G_{0}[i]\right|=\left|G_{1}\left[k_{p}\right]\right|
\end{array} \quad a\left(i, k_{1}\right)=0\right\}
\end{aligned}
$$

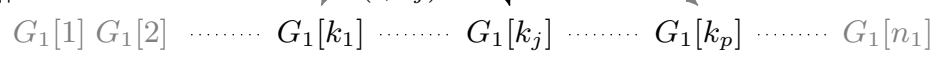

$$
\begin{aligned}
& \text { Genome } G_{1} \quad b_{1}\left(k_{1}\right) \in\{0,1\} \quad b_{1}\left(k_{j}\right)=1 \quad b_{1}\left(k_{p}\right) \in\{0,1\} \\
& b_{1}\left(k_{1}\right)+\ldots+b_{1}\left(k_{j}\right)+\ldots+b_{1}\left(k_{p}\right)=\min \left(\operatorname{occ}_{0}\left(\left|G_{0}[i]\right|\right), \operatorname{occ}_{1}\left(\left|G_{0}[i]\right|\right)\right.
\end{aligned}
$$

Fig. 2. Illustration of the constraints on variable $b_{0}(i), 1 \leq i \leq n_{0}$. If gene $G_{0}[i]$ appears in positions $k_{1}<k_{2}<\ldots<k_{p}$ in $G_{1}$ and gene $G_{0}[i]$ is mapped to gene $G_{1}\left[k_{j}\right]$ in the solution mapping, then (i) $a\left(i, k_{j}\right)=1$, i.e., gene $G_{0}[i]$ is mapped to gene $G_{1}\left[k_{j}\right]$, (ii) $a\left(i, k_{q}\right)=0$ for $1 \leq q \leq p$ and $q \neq j$, i.e., gene $G_{0}[i]$ is mapped to only one gene in $G_{1}$, (iii) $b_{0}(i)=1$, i.e., gene $G_{0}[i]$ is mapped to a gene of $G_{1}$ and (iv) $b_{1}\left(k_{j}\right)=1$, i.e., gene $G_{1}\left[k_{j}\right]$ is mapped to a gene of $G_{0}$. Observe that one may have in addition $b_{1}\left(k_{q}\right)=1$ for some $1 \leq q \leq p$ and $q \neq j$ if $\min \left(\operatorname{occ}_{0}\left(\left|G_{0}[i]\right|\right)\right.$, occ $1\left(\left|G_{0}[i]\right|\right) \geq 1$ (this observation is however no longer valid for the exemplar model).

Program Breakpoint-Maximum-Matching has $O\left(\left(n_{0} n_{1}\right)^{2}\right)$ constraints and $O\left(\left(n_{0} n_{1}\right)^{2}\right)$ variables, which could result in a time-consuming computation. Several simple rules have been used in order to speed-up the execution, some of which help to reduce the number of variables and constraints. They are discussed in the next subsection. 


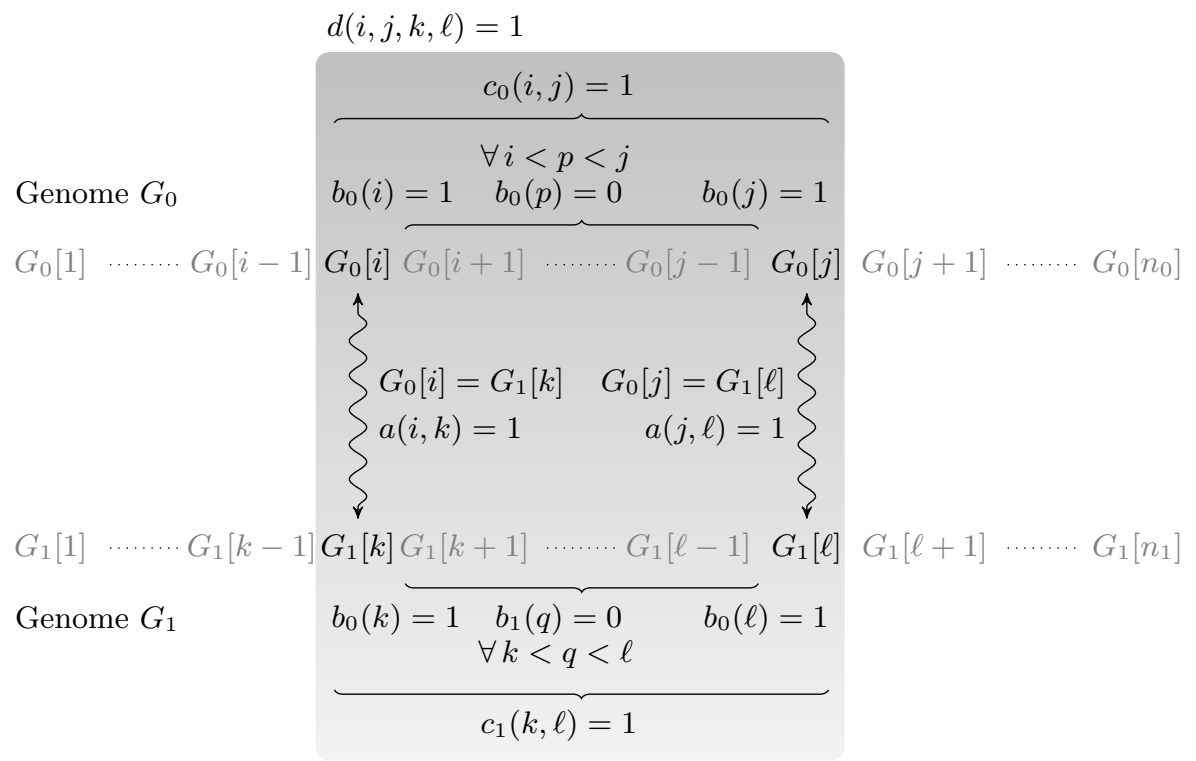

Fig. 3. Illustration of the constraints on variable $d(i, j, k, \ell), 1 \leq i<j \leq n_{0}$ and $1 \leq k<\ell \leq n_{1}$, for $G_{0}[i]=G_{1}[k]$ and $G_{0}[j]=G_{1}[\ell]$. The two genes $G_{0}[i]$ and $\left.G_{[j}\right]$ are adjacent according to a solution mapping if there exist two genes $G_{1}[k]$ and $G_{1}[\ell], G_{0}[i]=G_{1}[k]$ and $G_{0}[j]=G_{1}[\ell]$, such that (i) $G_{0}[i]$ is mapped to $G_{1}[k]$, i.e., $a(i, k)=1$, (ii) $G_{0}[j]$ is mapped to $G_{1}[\ell]$, i.e., $a(j, \ell)=1$, (iii) no gene between $G_{0}[i]$ and $G_{0}[j]$ is mapped to a gene of $G_{1}$, i.e., $c_{0}(i, j)=1$ and (iv) no gene between $G_{1}[k]$ and $G_{1}[\ell]$ is mapped to a gene of $G_{1}$, i.e., $c_{1}(k, \ell)=1$. The above situation reduces in our modelization to $d(i, j, k, \ell)=1$.

\subsection{Speeding-up the program}

We briefly describe in this section some rules for speeding-up the pseudo-boolean program.

Pre-processing the genomes. The genomes are pairwise pre-processed to delete all genes that do not appear in both genomes. For the exemplar model, consecutive occurrences of a gene (with the same sign) are reduced to only one occurrence to this gene. For the $\gamma$-proteobacteria benchmark set, the average size of a genome reduces from 3000 to 1300 .

Reducing the number of variables and constraints. Due to space constraints we only list few easy reduction rules. For non-duplicated genes, i.e., $\operatorname{occ}_{0}(g)=$ $\operatorname{occ}_{1}(g)=1$, the corresponding variable $a_{i, k}$ is set directly to 1 , as well as the two variables $b_{0}(i)$ and $b_{1}(k)$. Also, if two non-duplicated genes occur consecutively or in reverse order with opposite signs, the corresponding variable $d()$ is set directly to 1 and the related constraints are discarded. For the exemplar model, 
we must have exactly one occurrence of each gene in each genome, and hence if the same gene occurs, say in $G_{0}$, at positions $i$ and $j$, then the corresponding variable $d()$ is set directly to 0 and the related constraints are discarded. If for two genes, say occurring at positions $i$ and $j$ in $G_{0}$ and $k$ and $\ell$ in $G_{1}$, at least one gene occurring between position $i$ and $j$ in $G_{0}$ or $k$ and $\ell$ in $G_{1}$ must be saturated in any matching $\mathcal{M}$, then the corresponding variable $d(i, j, k, \ell)$ is set directly to 0 and the related constraints are discarded (details omitted).

Adding redundancy. While adding redundancy to a pseudo-boolean program is certainly useless from a correctness point of view, it can however have a major impact on the practical performance of the programs. For example, Program Breakpoint-Maximum-Matching contains some redundant constraints ((C.06), (C.08) and (C.10)) that significantly improved the running time of the program.

\section{Experimental results}

Thanks to the LPB program discussed previously, as well as formula (1), we are now able to determine the minimum number of breakpoints between pairs of genomes that contain duplicates. This minimum number of breakpoints will be computed according to the two above mentioned models, i.e. the exemplar and maximum matching models.

To this end, we used a dataset of $\gamma$-proteobacteria genomes, originally studied in [13], and exploited several times since then. This dataset is composed of twelve complete linear genomes of $\gamma$-Proteobacteria out of the thirteen originally studied in [13]. Indeed, the thirteenth genome ( V.cholerae) was not considered, since it is composed of two chromosomes, and hence does not fit in the model we considered here for representing genomes. More precisely, the dataset is composed of the genomes of the following species:

- Buchnera aphidicola APS (Baphi, Genbank accession number NC_002528),

- Escherichia coli K12 (Ecoli, NC_000913),

- Haemophilus influenzae Rd (Haein, NC_000907),

- Pseudomonas aeruginosa PA01 (Paeru, NC_002516),

- Pasteurella multocida Pm70 (Pmult, NC_002663),

- Salmonella typhimurium LT2 (Salty, NC_003197),

- Xanthomonas axonopodis pv. citri 306 (Xaxon, NC_003919),

- Xanthomonas campestris (Xcamp, NC_0 03902),

- Xylella fastidiosa 9a5c (Xfast, NC_002488),

- Yersinia pestis CO_92 (Ypest-C092, NC_003143),

- Yersinia pestis KIM5 P12 (Ypest-KIM, NC_004088) and

- Wigglesworthia glossinidia brevipalpis (Wglos, NC_004344).

The computation of a partition of the complete set of genes into gene families, where each family is supposed to represent a group of homologous genes, is taken from [5] (this partition was actually provided to these authors by Lerat [13]). It 
should be noted that in average, $11 \%$ of duplicated genes are present in these genomes.

The LPB engine is powered by minisat+ [12]. Computations were carried out on a Quadri Intel(R) Xeon(TM) CPU $3.00 \mathrm{GHz}$ with $16 \mathrm{~Gb}$ of memory running under Linux. Under the maximum matching model, minisat+ runs our program Breakpoint-Maximum-Matching (implemented using the speeding-up rules described in Section 3.3) in less than 10s for 56 out of the 66 possible pairs of genomes, and in several minutes for the remaining 10 pairs. The results are provided in Table 1.

The first conclusion that can be drawn from these results is the following: the pseudo-boolean approach we have considered here is a good approach for computing the minimum number of breakpoints for the maximum matching model, since all the results have been obtained within a few minutes. However, as already observed in [1] for maximizing the number of common intervals between two genomes, we notice that the exemplar model is the main bottleneck of our approach. Indeed, for the exemplar model, only 49 out of 66 (that is about $74 \%$ ) results have been obtained within a few minutes (we stopped the computation of the 17 remaining cases after a few days). We still have no formal explanation for this surprising and counter-intuitive fact. The 49 results we have obtained are given in Table 2 .

Besides the fact that computing the minimum number of breakpoints under the maximum matching model proves to be feasible under our pseudo-boolean approach, we find interesting to note that we have a sufficient number of results in both the maximum matching and the exemplar models to test the absolute accuracy of possible heuristics for these two problems. Indeed, if one wishes to obtain fast (though not optimal) results by using a given heuristic, it is relevant to know how tight this heuristic is. We carried out this study, focusing on two heuristics (one for the maximum matching model, the other for the exemplar model), that are both based on iteratively choosing a Longest Common Substring (LCS).

Maximum Matching Model. In [14], the authors introduced an heuristic that aimed at computing a matching between two genomes. This heuristic is a greedy algorithm based on the notion of $L C S$. Let $G_{0}$ and $G_{1}$ be two genomes: an $L C S$ of $\left(G_{0}, G_{1}\right)$ is a longest common word $S$ of $G_{0}$ and $G_{1}$, up to a complete reversal. The idea of the greedy algorithm is to match, at each iteration, all the genes that are in an $L C S$. If there are several $L C S$, one is chosen arbitrarily. In [1], we improved this heuristic in the following way: at each iteration, not only we match an $L C S$, but we also remove each unmatched gene of a genome, for which there is no unmatched gene of same family in the other genome. These rules imply that the resulting matching is a maximum matching. We call this heuristic IILCS_MM.

Exemplar Model. For the exemplar model, we use the same strategy (iteratively match the genes of an $L C S$ ), except that in this case we must make sure that only one gene from each family is matched on each genome. Therefore, at each 
iteration, and for each gene $\mathbf{g}$ present in the $L C S$ (and thus kept in the matching), we remove all the other occurrences of $\mathbf{g}$ in both genomes. Let us call this heuristic IILCS_EX.

We have tested both IILCS_MM and IILCS_EX under, respectively, the maximum matching and exemplar models. Current results are given in Tables 1 to 4 (see http://www.lri.fr/ thevenin/Breakpoint/\#Some_results for upto-date results). The two heuristics are quite fast and one can obtain all results for IILCS_MM and IILC_EX within 20 minutes on a regular desktop computer. For the maximum matching model, Heuristic IILCS_MM provides results that are on average $99.11 \%$ of the optimal number of breakpoints, ranging from $95.51 \%$ to $100 \%$. We actually note that in 14 out of the 66 cases, IILCS_MM returns the optimal value. Concerning IILCS_EX, the average, obtained over the 49 instances for which we know the optimal result, is $96.88 \%$, ranging from $94.38 \%$ to $99.10 \%$.

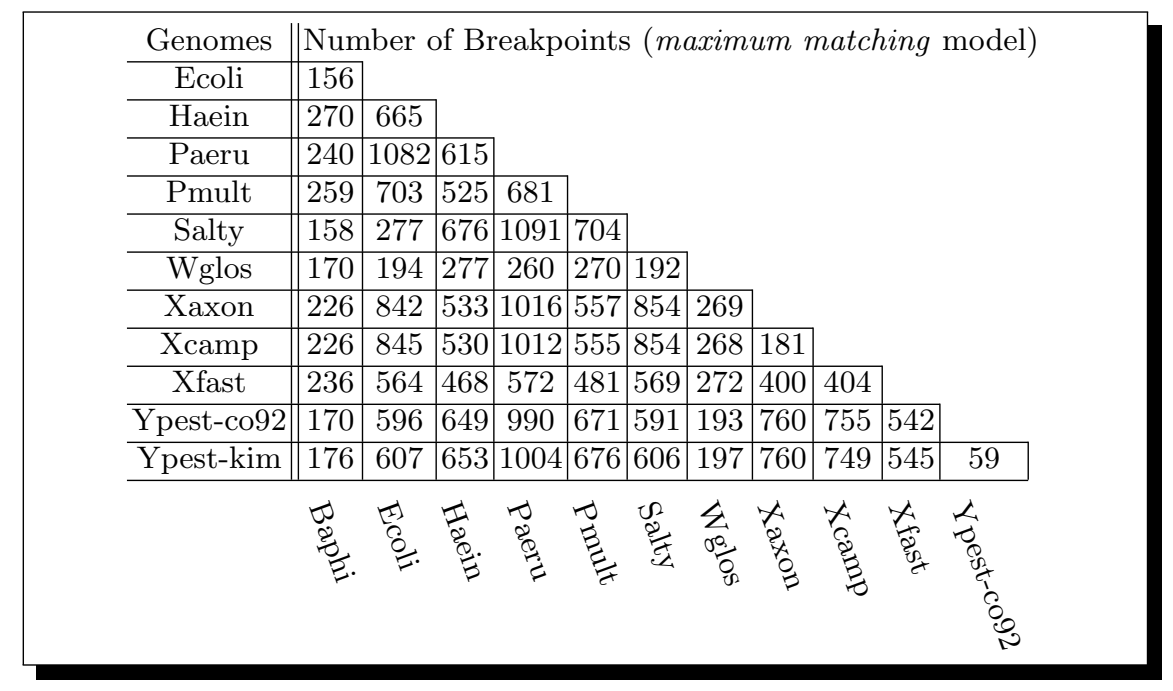

Table 1. Exact number of breakpoints for the maximum matching model

We thus conclude that both heuristics IILCS_MM and IILCS_EX, despite being extremely simple and fast, appear to be very good on the dataset we studied. In particular, for the exemplar model, since our pseudo-boolean approach seems to reach its limits for some instances, it could be convenient to compute those remaining instances using Heuristic IILCS_EX. 


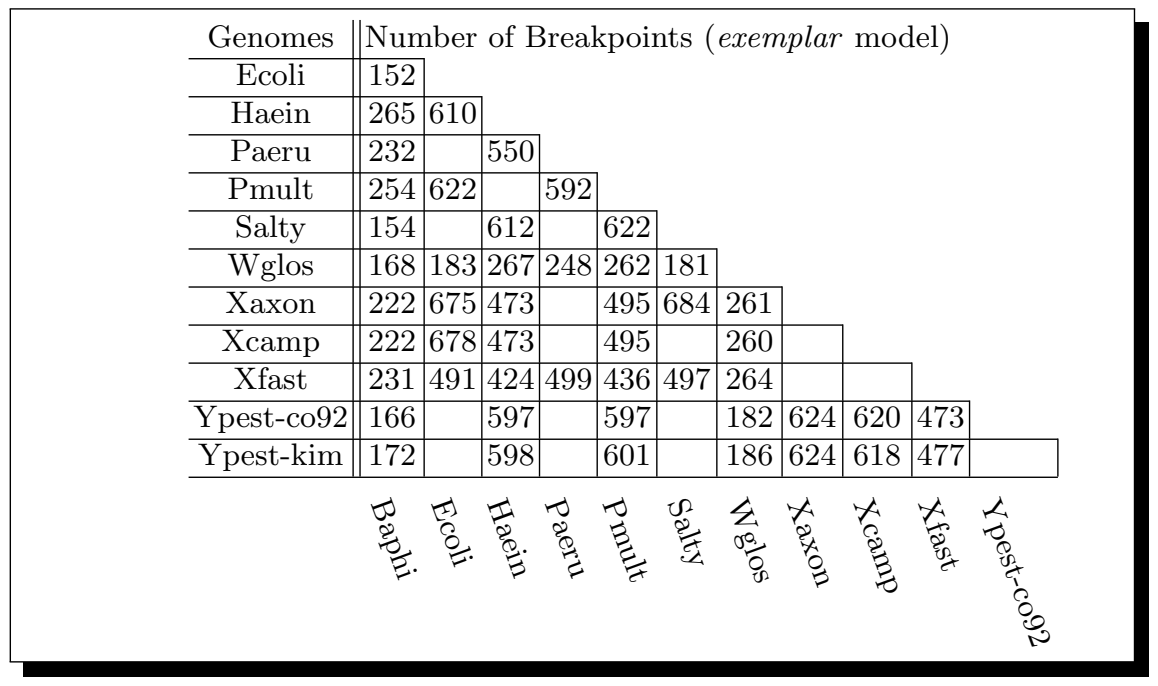

Table 2. Exact number of breakpoints for the exemplar model (49 instances out of 66)

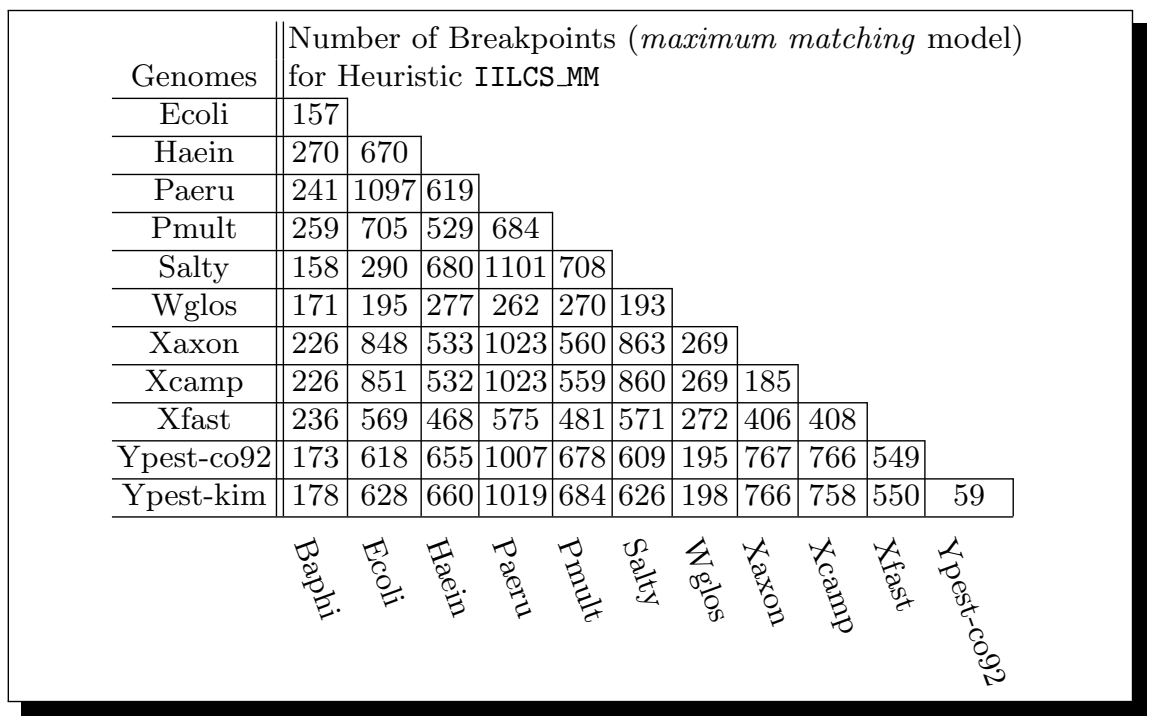

Table 3. Number of breakpoints for the maximum matching model by IILCS_MM

\section{Conclusion}

In this paper, we presented a method that helps speeding-up computations of exact results for comparing whole genomes containing duplicates. This method, 


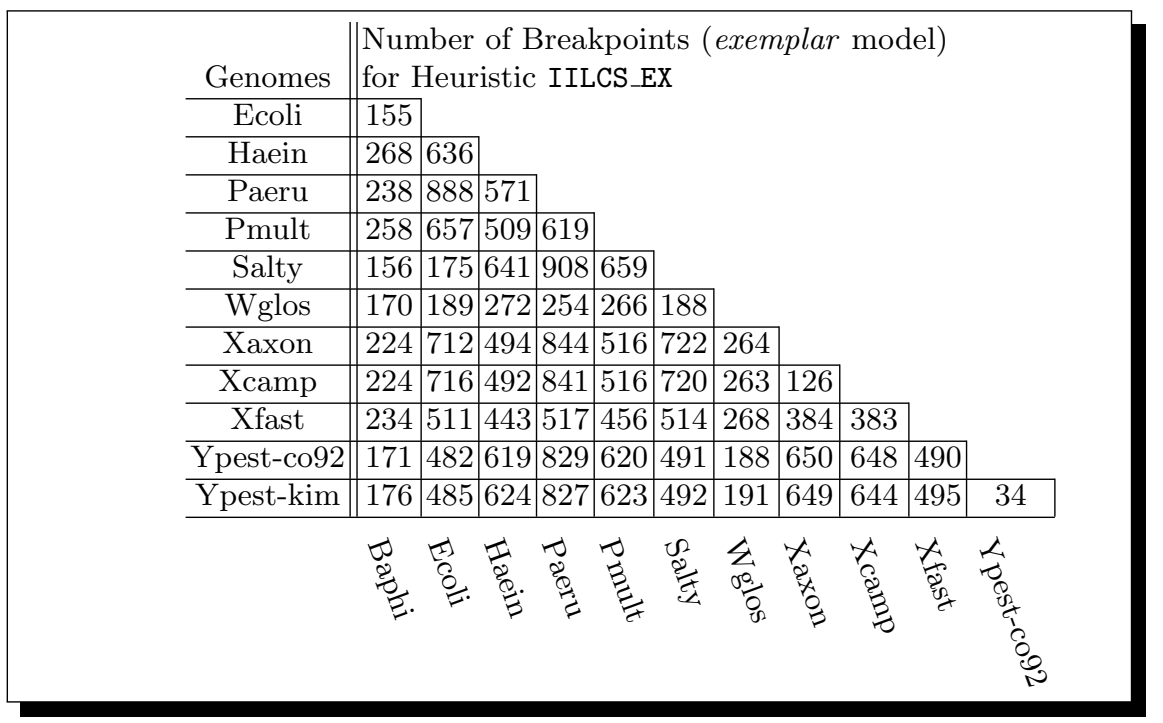

Table 4. Number of breakpoints for the exemplar model by IILCS_EX

which makes use of pseudo-boolean programming, has been introduced in [1] for computing the maximum number of common intervals between two genomes, and can be used for several (dis)similarity measures. In this paper, we used this method for computing the minimum number of breakpoints between two genomes, and developed pseudo-boolean programs for both the maximum matching and exemplar models. Experiments were undertaken on a dataset of $\gamma$ Proteobacteria, showing the validity of our approach, since all the results (resp. 49 results out of 66) have been obtained in a limited amount of time in the maximum matching model (resp. exemplar model). Moreover, these results allow us to state that both the IILCS_MM and the IILCS_EX heuristics provide excellent results on this dataset, hence showing their validity and robustness. On the whole, these preliminary results are very encouraging.

There is still a great amount of work to be done. For instance:

- Implementing and testing the maximum matching and the exemplar models, for several other (dis)similarity measures,

- For each case, determining strong and relevant rules for speeding-up the process by avoiding the generation of a large number clauses and variables (a pre-processing step that should not be underestimated),

- Obtaining exact results for each of these models and measures, and for different datasets, that could be later used as benchmarks in order to validate (or not) possible heuristics, and

- Implementing and testing an intermediate model between the maximum matching and the exemplar models, in which one must match at least one gene of each family in each genome. 


\section{References}

1. S. Angibaud, G. Fertin, I. Rusu, and S. Vialette. How pseudo-boolean programming can help genome rearrangement distance computation. In Springer, editor, Proc. 5th RECOMB Comparative Genomics Satellite Workshop, volume 4205 of LNBI, pages 75-86. springer, 2006.

2. S. Angibaud, G. Fertin, I. Rusu, and S. Vialette. A general framework for computing rearrangement distances between genomes with duplicates. Journal of Computational Biology, 14(4):379-393, 2007.

3. P. Barth. A Davis-Putnam based enumeration algorithm for linear pseudo-boolean optimization. Technical Report MPI-I-95-2-003, Max Planck Institut Informatik, 2005. 13 pages.

4. G. Blin, C. Chauve, and G. Fertin. The breakpoint distance for signed sequences. In Proc. 1st Algorithms and Computational Methods for Biochemical and Evolutionary Networks (CompBioNets), pages 3-16. KCL publications, 2004.

5. G. Blin, C. Chauve, and G. Fertin. Genes order and phylogenetic reconstruction: Application to $\gamma$-proteobacteria. In Proc. 3rd RECOMB Comparative Genomics Satellite Workshop, volume 3678 of LNBI, pages 11-20, 2005.

6. G. Blin and R. Rizzi. Conserved intervals distance computation between non-trivial genomes. In Proc. 11th Annual Int. Conference on Computing and Combinatorics (COCOON), volume 3595 of $L N C S$, pages 22-31, 2005.

7. G. Bourque, Y. Yacef, and N. El-Mabrouk. Maximizing synteny blocks to identify ancestral homologs. In Proc. 3rd RECOMB Comparative Genomics Satellite Workshop, volume 3678 of $L N B I$, pages 21-35, 2005.

8. D. Bryant. The complexity of calculating exemplar distances. In Comparative Genomics: Empirical and Analytical Approaches to Gene Order Dynamics, Map Alignment, and the Evolution of Gene Families, pages 207-212. Kluwer, 2000.

9. D. Chai and A. Kuehlmann. A fast pseudo-boolean constraint solver. In Proc. 40th ACM IEEE Conference on Design Automation, pages 830-835, 2003.

10. C. Chauve, G. Fertin, R. Rizzi, and S. Vialette. Genomes containing duplicates are hard to compare. In Proc Int. Workshop on Bioinformatics Research and Applications (IWBRA), volume 3992 of LNCS, pages 783-790, 2006.

11. X. Chen, J. Zheng, Z. Fu, P. Nan, Y. Zhong, S. Lonardi, and T. Jiang. Assignment of orthologous genes via genome rearrangement. IEEE/ACM Transactions on Computational Biology and Bioinformatics, 2(4):302-315, 2005.

12. N. Eén and N. Sörensson. Translating pseudo-boolean constraints into SAT. Journal on Satisfiability, Boolean Modeling and Computation, 2:1-26, 2006.

13. E. Lerat, V. Daubin, and N.A. Moran. From gene tree to organismal phylogeny in prokaryotes: the case of $\gamma$-proteobacteria. PLoS Biology, 1(1):101-109, 2003.

14. M. Marron, K.M. Swenson, and B.M.E. Moret. Genomic distances under deletions and insertions. Theoretical Computer Science, 325(3):347-360, 2004.

15. D. Sankoff. Genome rearrangement with gene families. Bioinformatics, 15(11):909917, 1999.

16. D. Sankoff and L. Haque. Power boosts for cluster tests. In Proc. 3rd RECOMB Comparative Genomics Satellite Workshop, volume 3678 of LNBI, pages 11-20, 2005.

17. A. Schrijver. Theory of Linear and Integer Programming. John Wiley and Sons, 1998.

18. H.M. Sheini and K.A. Sakallah. Pueblo: A hybrid pseudo-boolean SAT solver. Journal on Satisfiability, Boolean Modeling and Computation, 2:165-189, 2006. 